\title{
Immune Thrombocytopenic Purpura with Megaloblastic Anemia in Pregnancy
}

\author{
Beriwal S, ${ }^{1}$ Sabzposh N A, ${ }^{1}$ Anjum S, ${ }^{1}$ Ziyauddin F, ${ }^{1}$ Pratap R ${ }^{2}$ \\ ${ }^{1}$ Department of Obstetrics and Gynaecology, Jawaharlal Nehru Medical College, Aligarh Muslim University, India, \\ ${ }^{2}$ Department of Paediatrics, Era Medical College, Lucknow, India.
}

\begin{abstract}
Thrombocytopenia in pregnancy can be physiological or pathological. Causes can be differentiated based on period of gestation of presentation, degree of thrombocytopenia, clinical features and associated secondary causes. Thus, determining the significance of thrombocytopenia in a pregnant patient depends on the accurate identification of its underlying cause. A 21 years old primigravida was admitted to our hospital with very severe anemia and developed petechia all over her body. Her lab studies showed severe thrombocytopenia which responded to oral prednisolone whereby a diagnosis of thrombocytopenia was established. However, her general blood picture also showed megaloblastic anemia for which patient was investigated and treated. The aim of this report is to present a case of immune thrombocytopenic pregnancy along with megaloblastic anemia with pregnancy, responsive to steroid with risks to mother and fetus, and their concomitant management.
\end{abstract}

Keywords: ITP, megaloblastic anemia, petechia, prednisolone, pregnancy.

\section{INTRODUCTION}

Thrombocytopenia is second only to anemia as the most common hematological abnormality during pregnancy. ${ }^{1,2}$ Over recent times it has been commonly diagnosed because platelet counts are included in the automated blood cell counters. Causes of thrombocytopenia are numerous with few unique to pregnancy.

Most common cause in late pregnancy is gestational thrombocytopenia. One fifth cases are attributed to hypertensive disorders. ${ }^{1}$ ITP accounts for small number of cases although it is the most common cause of isolated thrombocytopenia in early pregnancy. ${ }^{1}$ Megaloblastic anemia is known to be commonly caused by folate or cobalamin deficiency. ${ }^{2}$ They are usually diagnosed with red cell indices showing raised mean corpuscular volumes and anemia not responding to iron therapy. Increased demand by the growing fetus along with a poor dietary intake is responsible for anemia in pregnancy. Hence, folate and iron supplements are advised in antenatal women.

\section{CORRESPONDENCE}

Dr Sri Devi Berniwal

Department of Obstetrics and Gynaecology,

Jawaharlal Nehru Medical College, Aligarh

Muslim University, India.

Email: sriiy2k@gmail.com
Few case reports have been published highlighting the association of megaloblastic anemia with thrombocytopenia. The article focuses on a case of ITP with megaloblastic anemia in pregnancy and its management.

\section{CASE}

A 20 yrs old primigravida with six months amenorrhea came to our hospital, on 30 April, 2012 with complaints of generalized body swelling for3-4 days associated with weakness, both gradual onset. There was no history of orthopnea, decreased urinary output, bleeding, fever, yellowish discoloration, backache, myalgia, stomatitis, dyspnea on exertion, any history of drug intake or blood transfusion. Patient had no similar history in the past, no chronic illness or any significant family history. Bowel and bladder habits were normal. Patient perceived fetal movements adequately.

On examination, she was severely pale and hadtachypnea. Her weight was $45 \mathrm{~kg}$. She had generalized swelling over her body with mild facial puffiness and pedal edema. Her vitals

The papers in this journal are published under the terms of the Creative Commons Attribution License. Users are allowed to read, download, copy, distribute, print, search, or link to the full texts of the articles in this journal without asking prior permission from the publisher or the author. 
were mostly stable with slightly raised respiratory rate.

There was no petechia or bruise, icterus, clubbing, koilonychias, brittle nails or any lymphadenopathy. Her systemic examination revealed normal findings. The uterus was 26 weeks size with perceivable fetal movements. Uterine tone was normal and there was no hepato splenomegaly.

Her baseline investigations revealed severe anemia with hemoglobin of $3.8 \mathrm{~g} / \mathrm{dl}$ and red cell indices showing macrocytosis. Her platelet count was $30,000 / \mu \mathrm{L}$ but reticulocytes were normal. The general blood picture also showed anisopoikilocytosis, microcytes, ovalocytes red cells along with numerous macrocytes and few target cells. Platelets were low on smear and there were no toxic granules. Blood coagulation profile remained normal.

To correct her anemia, one unit of packed RBC transfusion along with a single dose prophylactic antihelminthic was started.However, on the nextday petechia all over her body was noticed.But there were no bleeding from any other site. Repeat hematological investigations revealed persistent anemia and macrocytosis. Thrombocytopenia worsened further with plateletcount falling to $22000 / \mu \mathrm{L}$. However, coagulation profile remained normal. Folate and cobalamin levels for macrocytosis revealed very low levels.

For petechial rash assessment, dengue and malaria serology were done and were found negative. Bone marrow aspiration showed inactive megakaryocytes with increased cellularity of the bone marrow with both megaloblast and micronormoblasts and a reversed myeloid erythroid ratio. Giant metamyelocytes wAnd hypersegmented neutrophils were present. Pathologist concluded that both megaloblast and micronormoblasts seen with marked dyserythropoitic changes are suggestive of nutritional anemia. A provisional diagnosis of immune thrombocytopenia purpurawith megaloblastic anemia in pregnancy was made.

Tablet prednisolone $1 \mathrm{mg} / \mathrm{kg} /$ day on pre-pregnancy weight $(40 \mathrm{~kg})$ together with folic acid $5 \mathrm{mg}$ daily and hydroxycobalamin injections one milligram every alternate day for six doses were started. Patient showed a drastic improvement in her platelet count, which increased to $60,000 / \mu \mathrm{L}$ in two days and $1,22,000 / \mu \mathrm{L}$ in six days. Her hemoglobin levels also rapidly improved. Thus, a diagnosis of ITP with megaloblastic anemia in pregnancy was established.

\section{COMMENT}

Thrombocytopenia complicates $10 \%$ of all cases of pregnancy.1Even with its wide differential diagnosis, the cause of thrombocytopenia during pregnancy can usually be determined with a thorough history, physical examination, and directed laboratory studies. The challenge to the clinician is to weigh the risks of maternal and fetal bleeding complications against the benefits of diagnostic tests and interventions. ${ }^{2}$ The major differential diagnoses of thrombocytopenia in pregnancy are discussed below, highlighting the clinical and laboratory features useful in defining the cause of thrombocytopenia and ultimately narrowing the diagnosis.

The diagnosis of gestational thrombocytopenia is of exclusion and it is characterized by thrombocytopenia of mild to moderate degree, with platelet count less than $70,000 / \mu \mathrm{L}$, asymptomatic patients without history of bleeding; no evidence of pre-conception thrombocytopenia with a normal early gestation or preconception platelet count. The platelet count usually returns to normal within 2 to 12 weeks postpartum. ${ }^{2}$ Gestational or "Incidental" thrombocytopenia remains the most common cause of thrombocytopenia in pregnancy, accounting for approximately $75 \%$ of cases, and may be impossible to distinguish from mild ITP as platelet antibody are elevated in both disorders. ${ }^{1,9}$ However, gestational thrombocytopenia is not associated with adverse maternal or fetal outcomes. ${ }^{3}$

Immune thrombocytopenia purpura occurs in one or two of every 1,000 pregnancies, and accounts for $5 \%$ of cases. Despite its rarity compared to gestational thrombocytopenia, ITP is the most common cause of isolated thrombocytopenia in the first and early second trimesters. The pathophysiology of ITP in pregnancy is same as in non pregnant patient i.e. auto antibodies against platelet glycoprotein which are cleared by the reticuloendothelial system particularly the spleen. Though ITP accounts for only approximately onecase of thrombocytopenia per 1000 pregnancies and $5 \%$ of cases of pregnancy-associated thrombocytopenia, it is the most common cause of significant thrombocytopenia in the first trimester. ${ }^{1,2,6,7}$

ITP in women of child bearing age is usually insidious and variable. Although fluctuations in the platelet count may occur, the condition is not self limiting, and continuing thrombocytopenia is the usual course unlike ITP presentation in children which is acute and more self limiting. Chronic ITP may be asymptomatic and may be found by routine test performed during pregnancy.

Women with severe thrombocytopenia $<20 \times 10^{9} / \mathrm{L}$ are at a risk for spontaneous bleeding during antenatal as well as at delivery, and they generally require treatment. Women with platelet count less than $50 \times 10^{9} / \mathrm{L}$ may be at an increased risk of bleeding at delivery; therefore require treatment in late pregnancy to ensure safe platelet count at delivery in asymptomatic women. Both British and American guidelines suggest that a count $>50 \times 10^{9} / \mathrm{L}$ is safe for vaginal delivery. A platelet count $>80 \times 10^{9} / \mathrm{L}$ is required for administration of regional anesthesia. ${ }^{9}$ 
Fetal thrombocytopenia due to transfer of maternal antibodies is very low $(<2 \%)$ which have been clarified by large prospective studies conducted in 1990s. ${ }^{7,8}$

Management in prenatal period aims to treat maternal symptoms of hemorrhage at any stage of pregnancy and to achieve a safe platelet count for delivery.

Principal treatment options for the treatment of ITP in pregnancy include corticosteroids and high dose intravenous immunoglobulin. Corticosteroids are considered as the first line of therapy by most due to their efficacy and low cost. Prednisolone is started at dose of $1 \mathrm{mg} / \mathrm{kg} /$ day (based on non pregnant weight). A response is obtained in most patients and tapered gradually to achieve the minimum dose at which a safe platelet count is achieved. However other than osteoporosis, weight gain like in non pregnant women, corticosteroids also increase the risk of pregnancy induced hypertension and gestational diabetes and may promote premature rupture of membranes. Therefore, some experts suggest the high dose intravenous immunoglobulin $(2 \mathrm{mg} / \mathrm{kg})$ as first line therapy. However, transient effect which requires multiple courses make the treatment costly. Hence, they have been used in severe cases near to term for achieving minimal count for delivery.

Patients refractory to the above treatments are offered splenectomy in the second trimester with similar remission rate as in non pregnant patients of $75 \% .{ }^{10}$ Most recently anti-D is being used as the first or second line therapy in patients who are RhD positive in non splenectomised patient. ${ }^{9}$ The mechanism of action is not exactly known and thought to be by preferential removal of antibody coated RBC by the reticulothelial system. ${ }^{11}$ Refractory ITP istreated by immunosuppressants like azathioprine, rituximaband cyclosporine.

Megaloblastic anemia is a group of disorders characterized by the presence of distinct morphological appearances of the developing red cells in the bone marrow. The cause is usually deficiency of cobalamin (vitamin $B_{12}$ ) or folate. ${ }^{11}$ Many symptomless patients are detected through detectionof a raised mean corpuscular volume (MCV) on a routine blood count. The main clinical features are those of anemia. Anorexia is usually marked. Thrombocytopenia may cause bruising in severe cases and if associated with vitamin C deficiency.

Reduction in platelet count is widely recognized as part of dyshemopoetic pattern in megaloblastic anemia. However, to find a hemorrhagic diathesis is uncommon. Cases have been reported of hemorrhagic diathesis with megaloblastic anemia which have been successfully treated with cobalamin and folic acid to support this conception. ${ }^{12}$

Diagnosis is mostly laboratory based with typical symptoms in few patients. Oval macrocytes with considerable anisopoikilocytosis are the main features. The MCV is usually $>100 \mathrm{fL}$ unless causes of microcytosis e.g. iron deficiency, etc are present. Some of the neutrophils are hyper segmented (more than 5 nuclear lobes). There may be leucopenia but usually more than $1.5 \times 10^{9} / \mathrm{L}$; the platelet count may be moderately reduced, rarely $<40 \times 10^{9} / \mathrm{L}$; however the severity parallels the degree of anemia.

The bone marrow changes are of greatest significance. ${ }^{13}$ Bone marrow is hypercellular with accumulation of primitive cells. The cells are larger than normoblasts. Giant and abnormally shaped metamyelocytes and enlarged hyperploids megakaryocytes are characteristic. ${ }^{11}$ Serum levels are usually low (folate $<2 \mu \mathrm{g} / \mathrm{L}$; cobalamin $<100 \mathrm{ng} / \mathrm{L}$ ).

Treatment is to treat the cause of anemia with appropriate vitamin. Transfusion is usually unnecessary and if essential packed RBC should be given slowly. Platelet transfusion is not indicated as response to therapy is good.

\section{REFERENCES}

1. McCrae KR, Samuels $P$, Schreiber AD. Pregnancy associated thrombocytopenia: pathogenesis and management. Blood. 1992;80(11):2697-714.

2. Levy J, Murphy L. Thrombocytopenia in pregnancy: clinical review. J Am Board Fam Prac. 2002;15:290-7.

3. Stavrou E, McCrae K. Immune thrombocytopenic purpura. Hematol Oncol Clin North Am. 2009;23(6):1299-316.

4. Cines DB, Bussel JB, Liebman H. The ITP syndrome: pathogenic and clinical diversity. Blood. 2009;113(26):6511-21.

5. Provan D, Newland A. Idiopathic thrombocytopenic purpura in adults. J Pediatr Hematol Oncol. 2003(Suppl);1:S34-8.

6. Gill KK, Kelton JG. Management of idiopathic thrombocytopenic purpura in pregnancy. Semin Hematol. 2000;37:275-83.

7. Bell WR, Kickler TS. Thrombocytopenia in pregnancy. Rheum Dis Clin North Am. 1997;23:183-91.

8. Lescale KB, Eddleman KA, Cines DB, Samuels P, Lesser ML, McFarland $J G$, et al. Antiplatelet antibody testing in thrombocytopenic pregnant women. Am J Obstet Gynecol. 1996;174:1014-8.

9. Horn E, Kean L. Thrombocytopenia and bleeding disorders. High risk pregnancy expert consult. 4th ed. Missuori: Elsevier Saunders; 2011.

10. Gottlieb P, Axelsson O, Bakos O, Rastad J. Splenectomy during pregnancy: an option in the treatment of autoimmune thrombocytopenic purpura. Br J Obstet Gynaecol. 1999;106:373-5.

11. Fauci A, Braunwald E, Kasper D, Hauser S, Longo D, Jameson J. Harrison's principles of internal medicine. 17th ed. New York: McGraw Hill; 2008.

12. Smith $M$, Smith D, Fletcher $M$. Hemorrhage associated with thrombocytopenia in Megaloblastic anemia. BMJ. 1962;1(5283):9825 .

13. Trueman EG, Boyd J. Megaloblastic anemia of pregnancy with severe thrombocytopenic purpura: a case report and review of literature. Can Med Assoc J. 1959; 81(4):243-7. 